\title{
Author Index Volume 3
}

A Rudd, Murray, see Wang, Xiaowen

Appiah, Divine Odame, Forkuo, Eric Kwabena \& Bugri, John Tiah, Land Use Conversion Probabilities in a Peri-Urban District of Ghana

Ba Boxall, Alistair, see Wang, Xiaowen

Bugri, John Tiah, see Appiah, Divine Odame

Chao, Qingchen, see Zhou, Botao

Chao, Qingchen, see Li, Xiucang

Chen, Ying, Liu, Liyong \& Zhang, Ying, China's Urbanization and Carbon Emissions Peak

Chen, Ying, Post-2015 Development Agenda: From Concept to Practice

Chen, Zi, Liu, Changyi \& Qu, Shenning, China's Industrialization and the Pathway of Industrial $\mathrm{Co}_{2}$ Emissions

Chong, Zhaohui, see Qin, Chenglin

Cui, Tong, see Zhou, Bing

Feng, Zhaoxiang, see Liu, Zhe

Forkuo, Eric Kwabena, see Appiah, Divine Odame

Fu, Sha, Zou, Ji, Zhang, Xiaohua \& Qi, Yue, Review on the Latest Conclusions of Working Group III Contribution to the Fifth Assessment Report of the Intergovernmental Panel on Climate Change

Gu, Jintu, Luo, Jing \& Orderud, Geir Inge, Social Construction Based on Long-Distance Water Diversion Projects - A Case Study on the Yuqiao Reservoir in Tianjin

Gupta, Sunil, Sharma, Sanjay \& Saini, Randeep Singh, Evaluation of Stipulated Conditions Imposed at the Time of Grant of Environment Clearance From the Perspective of Implementation of Compliance-A Case Study for Construction Projects in India Howley, Peter, see Wang, Xiaowen

Huang, Lei, see Zhou, Botao

Huo, Ni, see Qin, Chenglin
4 (2015) 1550022

3 (2015) 1550026

4 (2015) 1550022

3 (2015) 1550026

1 (2015) 1550003

1 (2015) 1550004

3 (2015) 1550021

2 (2015) 1550015

3 (2015) 1550019

3 (2015) 1550024

2 (2015) 1550013

2 (2015) 1550010

3 (2015) 1550026

1 (2015) 1550005

2 (2015) 1550017

1 (2015) 1550006

4 (2015) 1550022

1 (2015) 1550003

3 (2015) 1550024 
Jiang, Tong, see Li, Xiucang

1 (2015) 1550004

Jiang, Wei, Could a Less Developed City Solve Its $\mathrm{Co}_{2}$ Emission Dilemma? Evidence From a Low Carbon Pilot City

1 (2015) 1550007

Li, Duo, see Zhou, Bing

2 (2015) 1550013

Li, Hongyu \& Li, Jie, Research on the Functional Dispersal and Population Governance for China's Megacities

Li, Jie, see Li, Hongyu

3 (2015) 1550018

3 (2015) 1550018

Li, Xiucang, Jiang, Tong, Chao, Qingchen, Xu, Hongmei, Yuan, Jiashuang \& Lin, Erda, The Core Conclusions and Interpretation of Working Group II Contribution to the Fifth Assessment Report of the Intergovernmental Panel on Climate Change

1 (2015) 1550004

Liang, Benfan, Pan, Jiahua, Zhang, Ying, Meng, Yanchun \& Zhu, Shouxian, China's Low-Carbon Urbanization Progress and Pathway

4 (2015) 1550028

Liang, Benfan, see Pan, Jiahua

2 (2015) 1550009

1 (2015) 1550004

3 (2015) 1550019

Liu, Changyi, see Chen, $\mathrm{Zi}$

2 (2015) 1550011

Liu, Jie, Liu, Changyi \& Wen, Yan, Reassessing Climate Change Impacts on Agriculture in China

2 (2015) 1550011

3 (2015) 1550021

Liu, Yong, Overall Coordination of Megacity Governance From a Regional Perspective

3 (2015) 1550020

Liu, Zhe, Feng, Zhaoxiang, Tian, Chunxiu, Zhang, Yiqiang \& Zhao, Wei, Co-Control of GHGs and Local Pollutants Under New Climate Regime

2 (2015) 1550010

Luo, Jing, see $\mathrm{Gu}$, Jintu

2 (2015) 1550017

Luo, Jing, see Tan, Hongze

Meng, Yanchun, see Liang, Benfan

Orderud, Geir Inge, see $\mathrm{Gu}$, Jintu

Orderud, Geir Inge, see Tan, Hongze

Pan, Jiahua, Environmental Sustainability of Mega-City:

Technology Possibility and Boundary Rigidity

3 (2015) 1550025

4 (2015) 1550028

2 (2015) 1550017

3 (2015) 1550025

4 (2015) 1550027

Pan, Jiahua, Liang, Benfan, Xiong, Na \& Qi, Guozhan, The Macro Path of China's Low-Carbon Urbanization

2 (2015) 1550009

Pan, Jiahua, see Liang, Benfan

4 (2015) 1550028

3 (2015) 1550025

Pan, Jiahua, see Tan, Hongze

2 (2015) 1550009

1 (2015) 1550005

Qi, Yue, see Fu, Sha

Qin, Chenglin, Huo, Ni \& Chong, Zhaohui, Intercity Rail Transit and Integrated Development of the Pearl River Delta Urban Cluster: Based on the Perspective of Network Analysis

3 (2015) 1550024 
Shan, Jingjing \& Wu, Zhanyun, Evaluation of China's Urban Healthy Development: Current Situation, Problems and Countermeasures

3 (2015) 1550023

1 (2015) 1550006

Tan, Hongze, Luo, Jing, Orderud, Geir Inge, Zheng, Yan \& Pan, Jiahua, The Pollution Caused by Protection: The Unintended Consequences of the Local Governance of the Urban Drinking Water Source Protection in Tianjin, China

3 (2015) 1550025

2 (2015) 1550010

Tian, Chunxiu, see Liu, Zhe

4 (2015) 1550033

Wang, Mou, Strategic and Economic Significances of the Construction of South Silk Road

2 (2015) 1550016

Wang, Xiaowen, Ba Boxall, Alistair, Howley, Peter \& A Rudd, Murray, Priority Research Questions on the Environmental Impacts of Pharmaceutical and Personal Care Products in China: Insights From Chinese Scientists

4 (2015) 1550022

Wei, Houkai, The Administrative Hierarchy and Growth of Urban Scale in China

1 (2015) 1550001

Wen, Yan, see Liu, Jie

2 (2015) 1550011

Wu, Baohua, Research on the Influential Factors of Rural Human Resources Development of Tianjin in the Context of New-Type Urbanization

Wu, Zhanyun, see Shan, Jingjing

$\mathrm{Xi}$, Jianchao, see Zhang, Ruiying

$\mathrm{Xi}$, Jianchao, see Zhang, Ruiying

Xiong, Na, see Pan, Jiahua

$\mathrm{Xu}$, Hongmei, see Li, Xiucang

Yang, Fating, Contemporary Construction of Ecological Civilization: From Ecological Crisis to Ecological Governance Ye, Jiang, Tentative Analysis of the Relationship Between the Implementation of the Coming Post-2015 SDGs and the Principle of $C B D R$

Yuan, Jiashuang, see Li, Xiucang

Zhang, Nan, see Zhang, Ruiying

Zhang, Nan, see Zhang, Ruiying

Zhang, Ruiying, Xi, Jianchao \& Zhang, Nan, The Comparative Study on Peasant Sustainable Livelihood Models in Rural Tourism Areas - A Case From Jixian, Tianjin

4 (2015) 1550029

3 (2015) 1550023

1 (2015) 1550008

2 (2015) 1550014

2 (2015) 1550009

1 (2015) 1550004

4 (2015) 1550030

2 (2015) 1550012

1 (2015) 1550004

1 (2015) 1550008

2 (2015) 1550014

2 (2015) 1550014 
Zhang, Ruiying, Xi, Jianchao \& Zhang, Nan, The Research on Peasant Sustainable Livelihood Models in Rural Tourism Areas Under Urbanization - A Case From Jixian, Tianjin

1 (2015) 1550008

4 (2015) 1550033

1 (2015) 1550005

4 (2015) 1550031

1 (2015) 1550002

3 (2015) 1550021

4 (2015) 1550028

2 (2015) 1550010

2 (2015) 1550010

3 (2015) 1550025

Zheng, Yan, see Tan, Hongze

Zheng, Yan \& Zhang, Xiaoyu, Risk Assessment of ClimateInduced Rural-Urban Migration: Case Study of Ningxia Based on Expert Judgment

4 (2015) 1550031

Zhou, Bing, Cui, Tong \& Li, Duo, Climate Monitoring and Formation Mechanism of Smog Pollution in China

2 (2015) 1550013

Zhou, Botao, Chao, Qingchen \& Huang, Lei, The Core

Conclusions and Interpretation of Working Group I

Contribution to the Fifth Assessment Report of the

Intergovernmental Panel on Climate Change

1 (2015) 1550003

4 (2015) 1550028

1 (2015) 1550005 